\title{
Closing the loop of the medication use process using electronic medication administration registration
}

- Bertil W. Lenderink and Toine C.G. Egberts

Pharm World Sci 2004; 26: 185-190.

(c) 2004 Kluwer Academic Publishers. Printed in the Netherlands.

B.W. Lenderink (correspondence, e-mail:

awlenderink@zamb.tsz.nl): Hospital Pharmacy

Midden-Brabant, TweeSteden Hospital and St. Elisabeth

Hospital, P.O. Box 90107, 5042 AD Tilburg, The Netherlands

T.C.G. Egberts: Department of Pharmacoepidemiology and

Pharmacotherapy, Utrecht Institute for Pharmaceutical

Sciences (UIPS), P.O. Box 80 082, 3508 TB Utrecht, The

Netherlands

\section{Key words}

Bedside pharmacy

Hospital pharmacy

Medication use process

Registration and documentation

The Netherlands

\section{Abstract}

Recent reports and studies of errors in the medication use process have raised the awareness of the threat to public health. An essential step in this multi-stage process is the actual administration of a medicine to the patient. The closed loop system is thought to be a way of preventing certain medication errors. Current information technology can facilitate this process. This article describes the way in which barcode technology is being used to facilitate medication administration registration on several wards in our hospital and nursing home.

Accepted January 2004 able studies originate from the USA, studies conducted in other countries such as the United Kingdom ${ }^{3}$ and the Netherlands ${ }^{4}$ have shown similar results. This strongly implies that medication errors are a major public health problem in many western as well as developing countries.

In light of these findings, we were therefore highly motivated to analyse the medication use process and to implement improvements, where necessary. The major conclusion from this particular analysis is that:

1) A medication order should contain all necessary items in such way that interpretation is only possible in a single manner and that there should not be more than one copy;

2) During the time of administration to the patient the uniquely identifiable medicine should be verified against the original medication order.

In addition, we concluded that these goals could only be achieved using electronic medication order entry and electronic registration of medication administration.

This article focuses on the most important, final step in the medication use process, viz., the administration of the medicine to the patient.

\section{Closed loop system}

The medication use process may involve up to 36 steps from the moment a doctor considers prescribing medication to the moment when this medication is actually administered or taken by the patient. Each step has the potential for error. The most error-free way to organise the medication distribution process is to design it as a 'closed loop system'. This means that the last steps of the first loop of the process are useful indicators in order to produce necessary information for the continuation of the process that has adopted this new information.

The essence of the closed loop system is that at the moment of medication administration, the medicine that is about to be given to the patient is verified against the medication order with respect to the necessary medicine characteristics (name, form, dose) and time. In addition, there must be verification that the specific medication is given to the right patient. Finally, the essential characteristics of the medication administration have to be documented in such a way that important elements, such as type of medication, patient, time of administration, and which person actually administers the drug, are retrievable afterwards. In this way, responsible health care providers will not only know what has been prescribed but also what type of medicines the patient has actually received and at what time. Guidelines concerning the in-patient medication use process, developed as a result of the renewed attention to medication errors, mandate a closed loop system ${ }^{5}$. Similarly, this approach is strongly recommended when transfusing blood to a patient ${ }^{6}$. 
In addition to the potential advantages of the closed loop system for direct patient care, the information derived from the medication administration registration can serve other purposes, such as quality indicators for evaluation of the medication distribution process, valid information to feed the logistic process, and medication utilisation review.

Although the basic idea of the closed loop is not new, current information technology enables us to actually identify potential errors arising from the medication distribution process. With respect to medication administration, automated bar coding seems to be the most feasible instrument to use at this moment. In our hospital each medicine is labelled with a specific barcode, which identifies the drug in question by name, strength, and dosage form (Figure 1a). Wherever possible, each unit of administration has an individual barcode; in other instances (e.g., multidose vials) the entire vial has a barcode. In addition, there is a barcode labelled on each wristband that in turn identifies the specific patient (Figure 1b).

At the time of the drug's administration the nurse takes the medication cart with the medicines for the current day to the ward(s) along with a tablet computer and a barcode scanner (Figure 2). The tablet PC is connected to the hospital's network through a wireless radio frequency connection. The nurse scans the barcode on the patient's wristband, after which the medication, which still has to be given that day to the same patient, is depicted on the screen of the PC (Figure 3a). Thereafter, the nurse scans the barcode on the medicine. The name of the medicine together with additional administration information is shown on the screen. When a medicine has been scanned for which a valid prescription is present, a subsequent administration can be scanned. However, when a medicine is scanned for which no medication order is available in the computer, a warning is given (Figure $3 b$ ).
The electronic medical record contains historical information concerning the medication administered with several details for each administration (Figures 4 and 5).

\section{Considerations}

The above process, ideal as it may be, may not be feasible or even necessary in all situations, though. For example:

\section{Medication order entry versus medication administration registration}

- In the emergency department the timing of actions is extremely important and circumstances are occasionally such that there is no time to register prescriptions before the medicines are administered. At those moments the system should aid the administrator (i.e., healthcare professional) to at least provide and administer the ordered medication with some essential checks, such as dose, known allergy and interaction with other medicines already taken by or given to the patient. The ordering physician should be able to authorise the administrations afterwards and, if necessary, continue the pharmacotherapy that has been initiated.

These circumstances also apply to operating theatres and intensive care units.

- In psychiatric wards some patients find it difficult to wear wristbands with their name and barcode. An alternative means should be invented to identify such patients.

- Regularly used medicines such as infusion fluids (e.g., normal saline or dextrose 5\%) or certain indifferent ointments and creams might not be classified as dangerous and therefore are not considered candidates for the closed loop system. a) patient barcoded

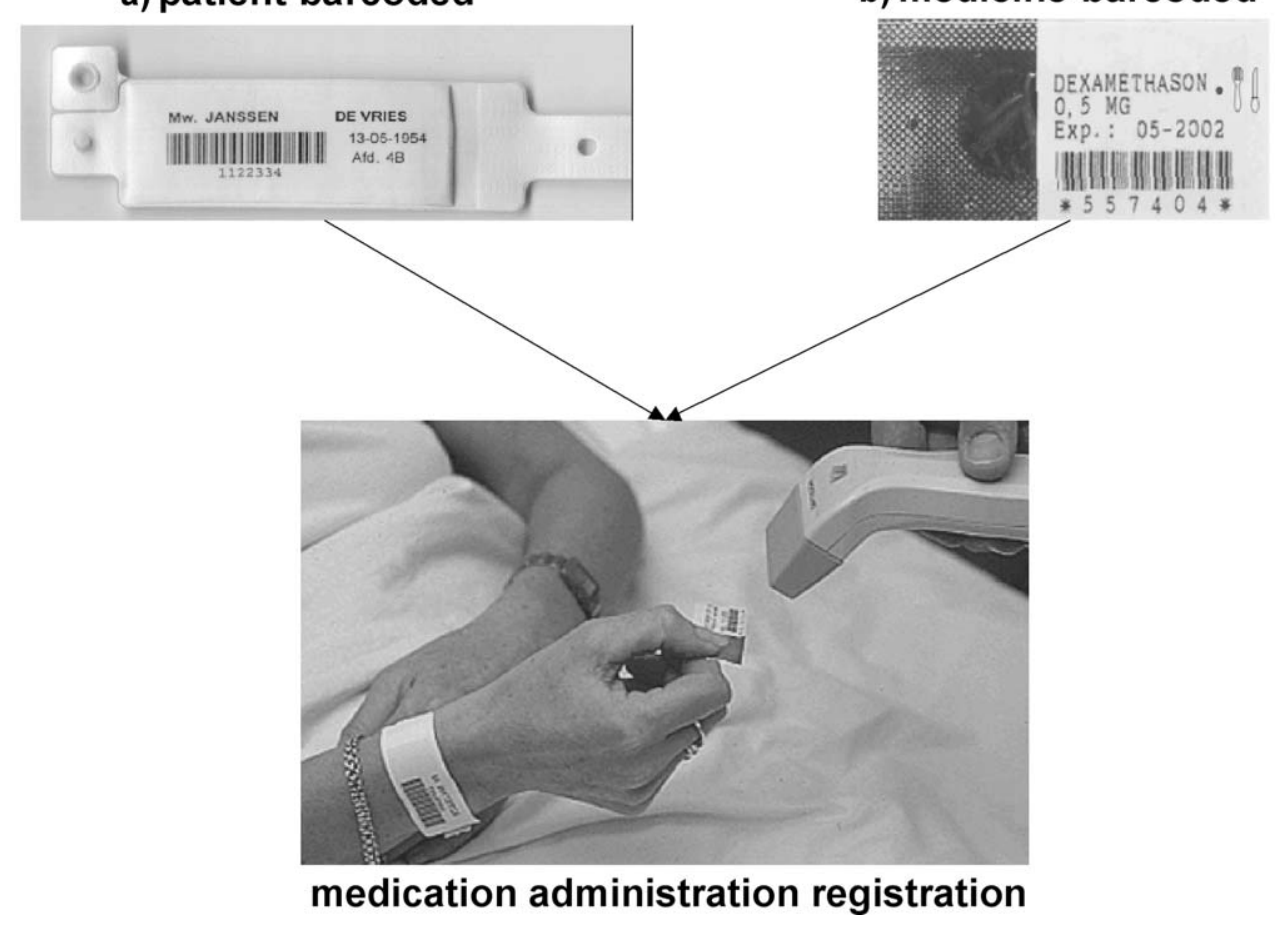

Figure 1 Barcoding of drugs and patients. 
- Certain medication regimens are frequently modified, leading to an increase in workload to ensure that every modification is entered into the computerised physician order entry system. Most often protocols are designed that provide the nurse instructions or scenarios for when a dose may be changed. Some countries are even on the verge of introducing nurse or pharmacist prescribing. Registration of such dosage modifications also requires the underlying reasons for the change and is becoming an important step in the medication administration process. This situation is certainly possible with analgesics or use of oral anti-coagulants, for example.

- The administration of radiology contrast media is another example of medication use that is protocolised in a similar fashion, meaning that that the sole registration of the administration without a specific prescription will suffice.

- Another therapeutic category requiring special attention is blood products. In order to ensure adequate haemovigilance, there are in some countries a number of legal instructions which demand the registration not only of which product has been given to the patient but also from which batch it originated 7,8 .

\section{Positive versus negative registration}

When it is decided that medication administration registration is useful, it can be achieved in different ways: - By registering what is actually given to the patient positive registration, as described above;

- By registering what has not been given to a patient in comparison with the prescription - negative registration.

In the latter case, negative registration of drug administration means that every dispensed medicine was administered as it was prescribed, except for those that are still in the patient's drawer the next time round. This may have the disadvantage that one is less certain

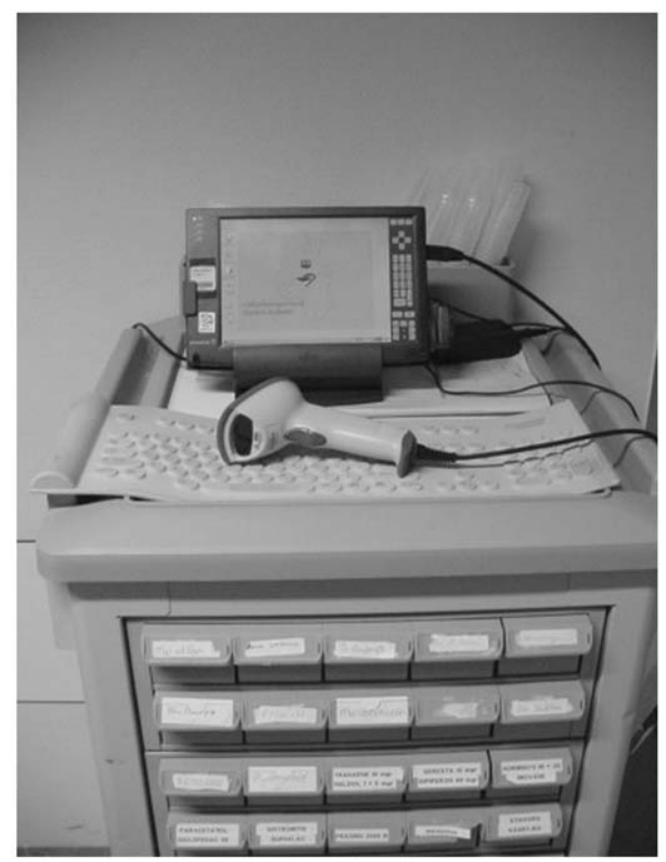

Figure 2 Medication cart with tablet PC, flexible keybord and barcode scanner. whether the right patient got the medication. On the other hand, an advantage of this method is that the process is much less time consuming. There are some studies, however, that describe 10-15\% returned doses in an average ward, and even higher percentages in a surgical ward because of 'sobriety' policies.

Other problems with negative medication administration registration are: the difficulty of acquiring information about extra doses of ordered medicines or about single, incidental administrations of unordered medications. Their number may be $2-5 \%$ of all administrations ${ }^{9}$.

\section{Location of registration}

Positive medication administration registration is probably best performed at the place of origin, i.e., at the bedside. It has the advantage that it is more difficult to forget what has actually occurred during administration of the drug(s), simply because there is no interference between the administration and the registration. A disadvantage is that one needs mobile registration equipment (no need for commas), which might pose a problem, particularly when one has to transport, for example, a flask, tubing and an infusion bag together with some oral liquids and tablets/capsules all for the same patient. Bringing extra non-sterile computer equipment into an operating theatre or emergency department is sometimes difficult.

It is possible that a positive medication administration registration system can be implemented using a combined approach. For example, by administering some of the $\operatorname{drug}(s)$ at the bedside and some in the ward. Neglecting the registration of drug administration at the bedside may lead to the problem of 'undercharting'. A situation where this could actually occur is when the administering nurse is distracted after the administration and forgets the registration. It also increases the risk of administering medicines to the wrong patient.

If the introduction or use of mobile equipment is a problem, positive medication administration registration at the ward is always preferred over negative registration in the ward and certainly over registration inside the pharmacy department after return of the 'empty' medication carts.

Modern logistic systems, such as Pyxis, Hydra and Omnicell, use a method which diverges from the positive manner of drug registration. These systems do not favour an optimal implementation of the medication process as the patient is not personally identified, except as a line on the computer screen. In addition, medicines are not identified, reference being made only to bins in a drawer.

In the Netherlands experiments are currently being conducted with a computerised mobile stock cabinet, which is used during a medication ward round with drawers and ward drug formularies medicines (bedside assorted picking).

\section{Conclusion}

It is likely that adopting several approaches can reduce the number of errors in the medication distribution systems. One way is to educate the employees who work within the system, since 'to err is human'?

In addition, the use of modern technology, such as bar coding and scanning, can reduce the number of 
errors made when inserting medication use data into a computer system which eventually transforms the distribution process into a closed loop system.

After acquiring knowledge and data from older and newer studies, we should strive to introduce these new techniques as soon as possible simply because many patients can be saved from serious harm whilst admitted to the hospital.

From these newer systems we will automatically obtain an abundance of information, including error re-

a)

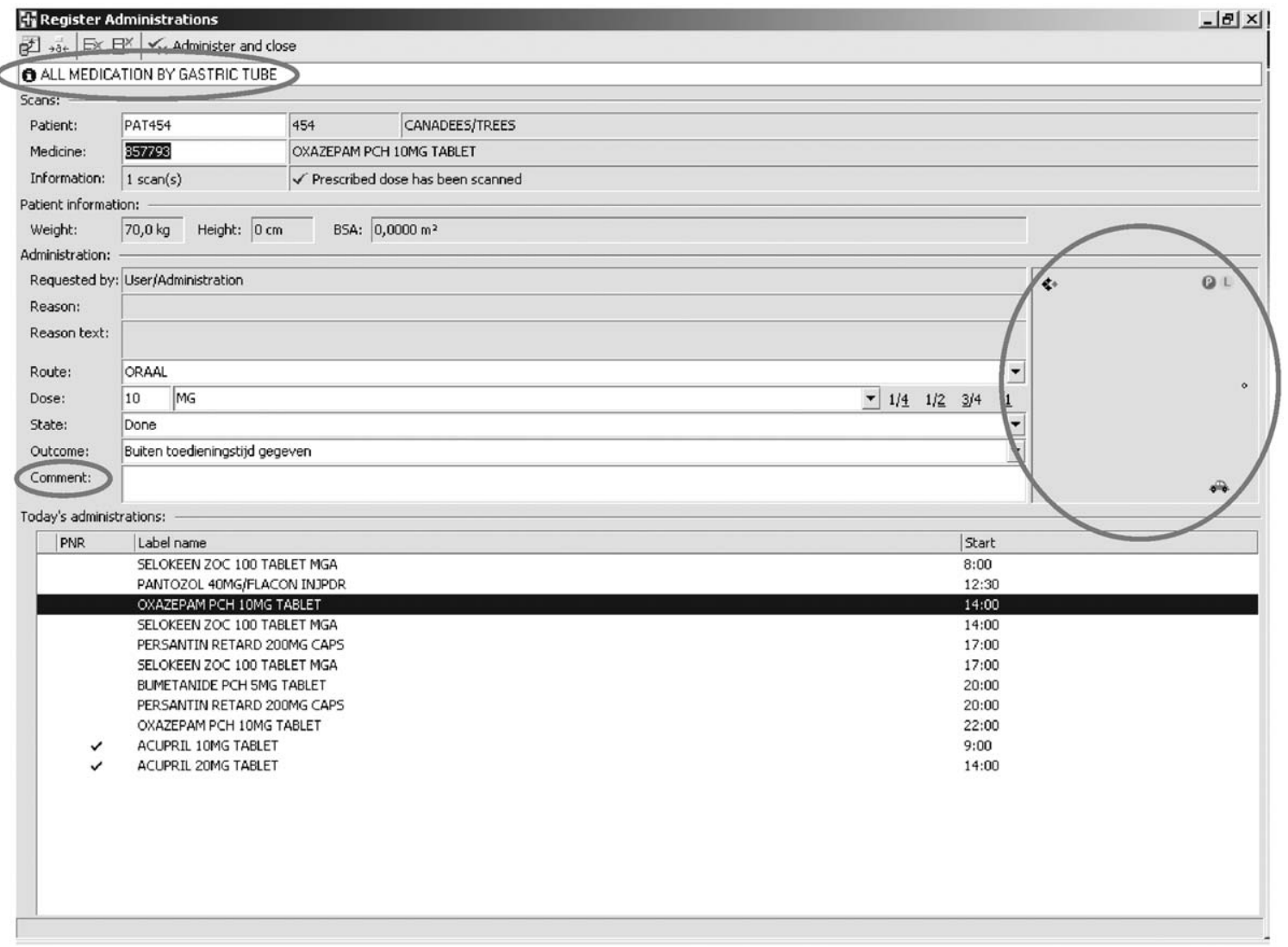

b)

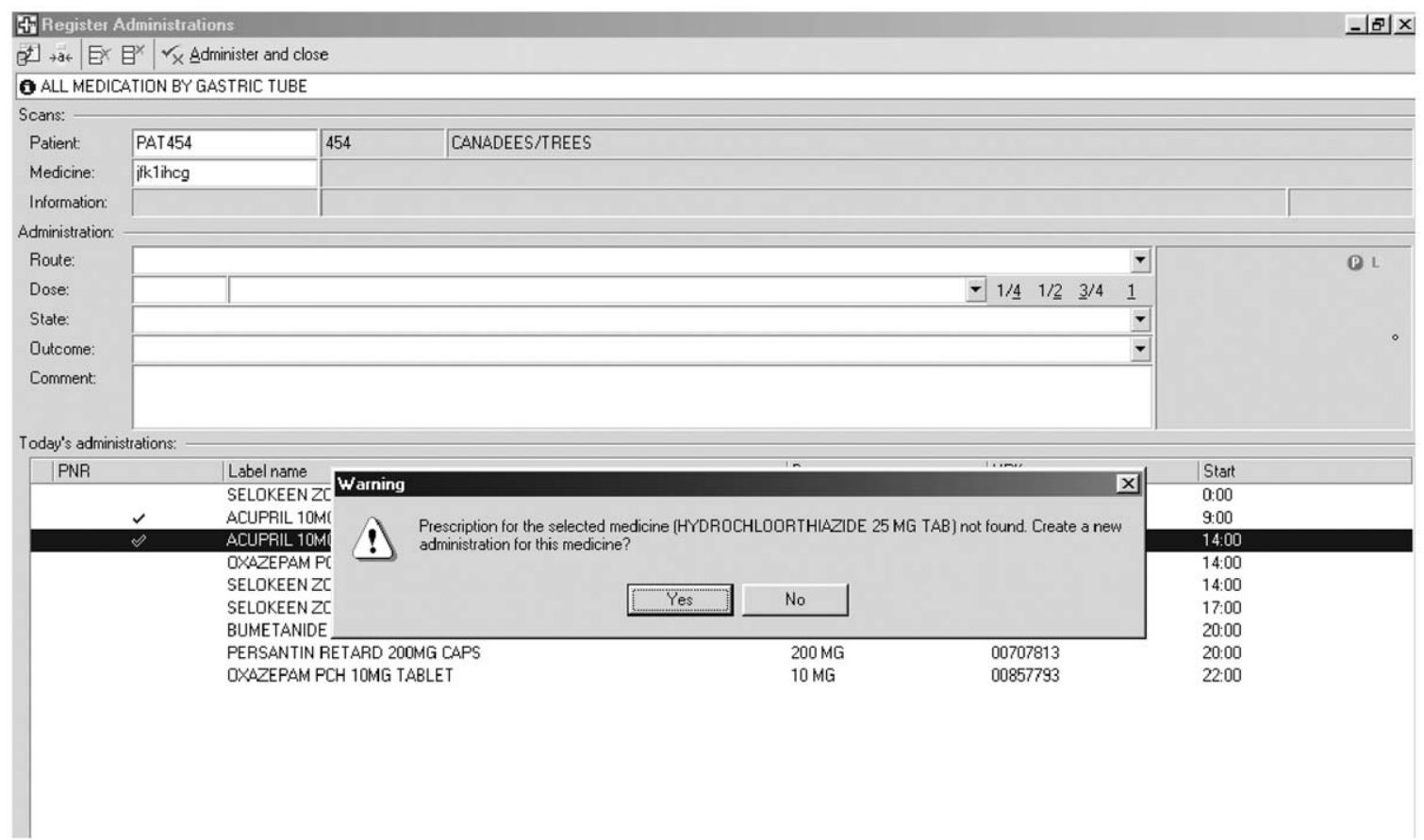

Figure 3 Some examples of screens that help the nurse to administer the right medication to the right patient in the right way at the right time. (a) After scanning the wristband and the medicine the nurse can enter comments about the administration if necessary. She/he is also warned about interactions by means of a picture(circled figure) and how to administer the medicine (e.g., all medications by gastric tube). (b) When a nurse scans and tries to administer a non-prescribed medicine she/he is warned. 


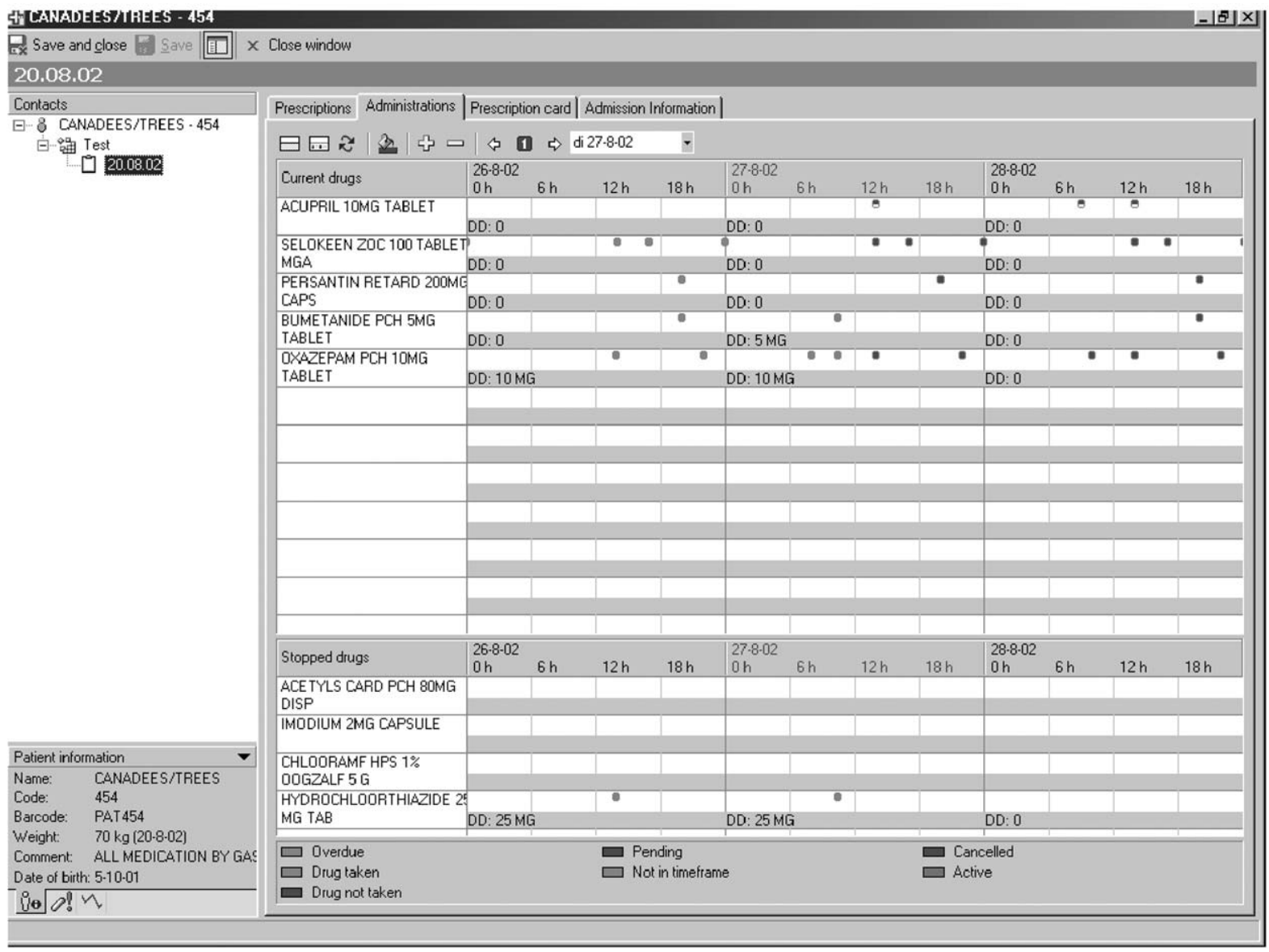

Figure 4 After prescribing and scanning, this screen presents a complete overview of what happened with the orders.

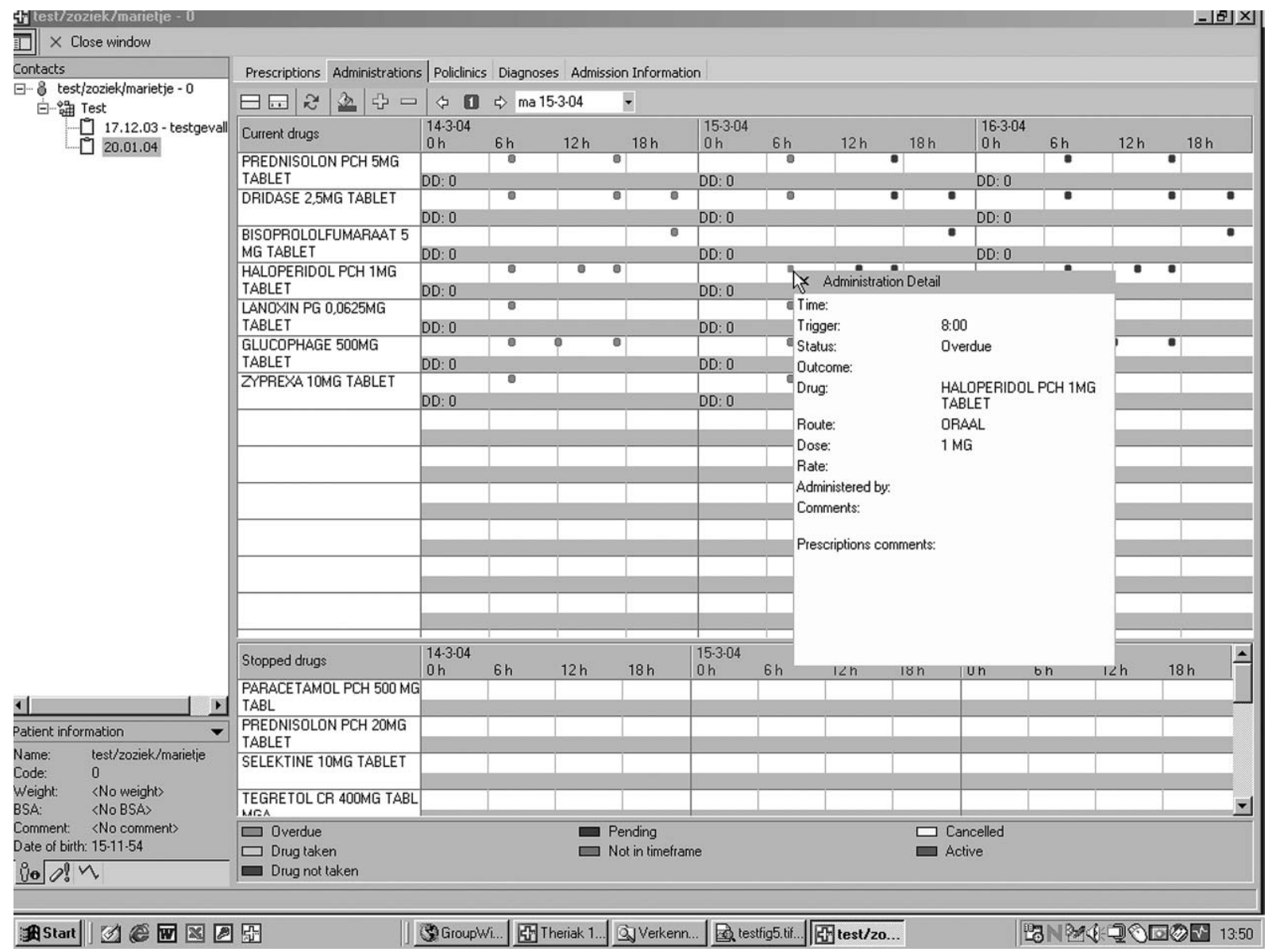

Figure 5 Details on the administration can also be found in the same screen. 
ports, for example, which will make quality improving interventions possible. On the other hand, this type of information should not be used to blame those health care professionals who had caused these automatically registered errors.

\section{Acknowledgement}

Software development and process implementation have been made possible by means of a grant from the European Union: IST 2000-23260 PHARMA.

\section{References}

1 Barker KN, McConnell WE. Detecting errors in hospitals. Am J Hosp Pharm 1962; 19: 361-9.

2 Kohn LT, Corrigan JM, Donaldson MS, editors. To err is human. Building a safer health system. Institute of Medicine. Washington, DC: National Academy Press, 1999
3 The Audit Commission London. A spoonful of sugar. Medicines management in NHS Hospitals. Portsmouth, UK: Holbrooks Printers, 2001

$4 \mathrm{Vd}$ Bemt PMLA. Drug Safety in Hospitalised Patients. Thesis. Groningen University, Groningen, The Netherlands, 2002.

5 The National Coordinating Council for Medication Error Reporting and Prevention (NCC MERP). www.nccmerp.org (15 August 2002).

6 Regan F, Taylor C. Recent developments. Blood transfusion medicine. BMJ 2002; 325: 143-7.

7 Love EM, Soldan K. Serious hazards of transfusion. Summary of annual report 1999-2000.

8 Haas FJLM, Harvey MS, Hazenberg CAM, Idema RN, van Voorst tot Voorst E. De transfusieketen in ziekenhuizen: aanbevelingen voor kwaliteitsborging. Ned Tijdschr Klin Chem 2001; 26: $203-$ 13.

9 Max BE, Itokazu G, Danzinger LH, Weinstein RA. Assessing unit dose discrepancies. Am J Health Syst Pharm 2002; 59: 856-8. 